REVISTA DE GESTÃO E SECRETARIADO

MANAGEMENT AND ADMINISTRATIVE

PROFESSIONAL REVIEW

ISSN: 2178-9010
Revista GeSec

São Paulo, SP, Brasil

v. 12 , n. 2, p. 327-352

maio /ago. 2021

DOI: http://dx.doi.org/10.7769/gesec.v12i2.1203

\title{
Uma medida da lealdade ao bairro: validade de construto, convergente e discriminativa em moradores em distintas zonas urbanas na cidade de Natal-RN, Brasil ${ }^{1}$
}

\section{A measure of loyalty to the neighborhood: construct validity, convergent} and discriminative in residents in different urban areas in the city of NatalRN, Brazil

\author{
Sheila Trícia Guedes Pastana ${ }^{2}$ \\ Nilton Soares Formiga ${ }^{3}$
}

\section{Resumo}

O presente artigo tem como objetivo desenvolver e validar uma medida de escala de lealdade ao bairro, a partir da perspectiva de indivíduos que habitam nas áreas sul e leste da cidade de Natal-RN. Tem como direção conceitual a compreensão das dimensões da qualidade de serviços intraurbanos, atinentes a perspectiva da qualidade de vida urbana, que relacionam estudos que advêm da sustentabilidade urbana, logística da cidade, mobilidade sustentável, cidades inteligentes, planejamento, gestão urbana e satisfação residencial. Concernir essas complexas inter-relações acima descritas consolida o entendimento deste estudo. Em função disso, propõe-se uma ferramenta de mensuração da lealdade de moradores para com seus bairros, possibilitando insights que poderão direcionar a tomada de decisão por parte dos gestores, favorecendo bairros mais atraentes, acolhedores, o bem-estar das pessoas e investimentos públicos e privados. Participaram do estudo 545 respondentes, homens e mulheres, acima de 18 anos, distribuídos em duas zonas residenciais, sendo 54\% da zona sul e $46 \%$ da zona leste. Através da coleta de dados do tipo Survey, ao finalizá-la, realizou-se estatísticas descritivas, análise fatorial exploratória e confirmatória, bem como, Anova. Quanto aos resultados, os testes psicométricos deram robustez e confiabilidade a este estudo,

\footnotetext{
1 O presente trabalho foi realizado com apoio da Coordenação de Aperfeiçoamento de Pessoal de Nível Superior - Brasil (CAPES) - Código de Financiamento 001.

2 Doutora em Administração, Docente da Universidade Federal do Amapá (UNIFAP).

${ }^{3}$ Doutora em Psicologia Social, Docente no Programa de Pós-graduação em Psicologia Organizacional e do Trabalho da Universidade Potiguar (UnP).
} 
evidenciando que a escala Lealdade ao Bairro (LB) é segura às referidas mensurações, consistente e fidedigna a uma estrutura fatorial de um único fator e que não houve diferença entre as zonas urbanas pesquisadas, comprovando com isso, o mesmo nível de lealdade.

Palavras-Chave: Gestão dos bairros. Gestão urbana. Lealdade. Escala de lealdade ao bairro. Qualidade de vida.

\begin{abstract}
This article aims to develop and validate a scale measure of loyalty to the neighborhood, from the perspective of individuals who live in the south and east areas of the city of Natal-RN. The conceptual direction is to understand the dimensions of the quality of intra-urban services, related to the perspective of the quality of urban life, which relate studies that come from urban sustainability, city logistics, sustainable mobility, smart cities, planning, urban management, and residential satisfaction. Concerning these complex interrelations described above consolidate the understanding of this study. As a result, we propose a tool for measuring the loyalty of residents to their neighborhoods, providing insights that can guide decision making by managers, favoring more attractive, welcoming neighborhoods, people's well-being, and public investments. and private. 545 respondents, male and female, over 18 years old, participated in the study, distributed in two residential areas, $54 \%$ from the south and $46 \%$ from the east. Through the collection of data of the Survey type, at the end of it, descriptive statistics, exploratory and confirmatory factor analysis were carried out, as well as Anova. As for the results, the psychometric tests gave robustness and reliability to this study, showing that the Loyalty to the Neighborhood scale (LB) is safe to the referred measurements, consistent and reliable to a factorial structure of a single factor and that there was no difference between the zones. urban areas surveyed, thereby proving the same level of loyalty.
\end{abstract}

Keywords: Neighborhood management. Urban management. Loyalty. Scale of loyalty to the neighborhood. Quality of life.

\title{
Introdução
}

A compreensão das questões urbanas tem sua dinâmica envolvida por complexas interrelações, e na perspectiva das percepções de satisfação, apego e lealdade com o lugar de 
convívio, percebe-se que a qualidade dos espaços urbanos impacta diretamente na qualidade de vida nas cidades, podendo influenciar na permanência ou mudança do ambiente de moradia.

A construção desse estudo partiu da teoria da qualidade de serviços que estabelece uma relação causal entre qualidade, satisfação e lealdade. Nesse entendimento, a qualidade tem uma relação direta e positiva com a satisfação e esta tem relação direta e positiva com a lealdade (Cronin Jr \& Taylor, 1992; Fitzsimmons \& Fitzsimmons, 2014; Gronroos, 1984; Parasuraman, Zeithaml, \& Berry, 1985). Qualidade possui característica multidimensional, já a satisfação e a lealdade representam um estado psicológico que recebe influência da qualidade.

Em relação à qualidade dos serviços do bairro, especialistas apontam que fatores ambientais, características físicas do bairro, da vizinhança, infraestrutura, o acesso aos serviços públicos e privados, arborização viária, áreas de lazer e a percepção subjetiva e objetiva do seu entorno têm influência na qualidade de vida das pessoas, ou seja, que o grau de satisfação com o espaço habitacional exerce influência na vida das pessoas de forma geral (Bougouffa \& Permana, 2018; Elvas \& Moniz, 2010; Galloway, 2006; Grogan-kaylor et al., 2006; Hadavi, Kaplan, \& Hunter, 2018; Ibem \& Aduwo, 2013; Khaef \& Zebardast, 2015; R. Marans \& Rodgers, 1975; R. W. Marans, 2012; Momtaz, Fallahi, \& Delbari, 2018; Riecken, Shemwell, \& Yavas, 2000; Sirgy \& Cornwell, 2002; Yang, 2008)

De forma geral, a lealdade origina-se da relação da qualidade do serviço e da satisfação, e ambas exercem um impacto direto e positivo sobre a lealdade das pessoas. Essa fidelização faz com que o indivíduo desenvolva um compromisso consistente e queira repetir a experiência (Chang \& Thai, 2016). Bem como, de acordo com Chen e Cheng (2012), a satisfação e percepção de valor tem impacto direto sobre a lealdade do indivíduo, pois este construto tem componentes atitudinais e comportamentais, que levam a um compromisso mais constante da pessoa que assume esta condição (Caceres \& Paparoidamis, 2007).

Desta maneira, a fim de verificar a existência desse fenômeno, com base numa escala de medidas, buscou-se na base de dados Scopus, estudos que apresentassem escalas que atendessem ao objetivo desta pesquisa. Devido a inexistência de uma escala específica, bem como, com base na afirmação de Smrke (2018), para o qual, existe uma falta generalizada de instrumentos adequadamente construídos e validados de forma confiável para a verificação a lealdade ao bairro na percepção de seus moradores.

De acordo com uma revisão sistemática da literatura, a maioria dos instrumentos não são publicados (Smrke, Blenkuš, \& Sočan, 2018). Com isso, não é possível acompanhar o desenvolvimento de uma avaliação e explicação segura a respeito do fenômeno abordado neste estudo, bem como, propor um modelo teórico com uma estrutura fatorial mais robusta que 
contemplasse a definição da lealdade ao bairro. Portanto, dada a inexistência de outro instrumento seguro e em função da relevância dos estudos intraurbanos aliados à lealdade, este artigo tem como objetivo, o desenvolvimento e validação de conteúdo e construto de uma medida de escala de lealdade ao bairro, a partir da perspectiva de pessoas que habitam as áreas sul e leste na cidade de Natal-RN.

\section{Lealdade ao Bairro e sua perspectiva conceitual}

Há estudos que abordam a relação causal entre qualidade do serviço e satisfação como antecedentes da lealdade e, é possível associar à luz da qualidade dos serviços a essência desse modelo conceitual, onde: Qualidade; Satisfação e Lealdade, representam uma sequência lógica nessa interação. Cabe ressalta que o termo lealdade também é abordado na literatura como sinônimo de fidelidade, preferência, apego, gratificação e prazer (Ahrholdt, Gudergan, \& Ringle, 2016; Amerigo \& Aragones, 1997; Caceres \& Paparoidamis, 2007; Chang \& Thai, 2016; Chen \& Cheng, 2012; Deng, Lu, Kee, \& Zhang, 2010; Fornara, Bonaiuto, \& Bonnes, 2010; Harris \& Goode, 2004; Kassim \& Abdullah, 2010; Lai, Grif, \& Babin, 2009; Lee, Lee, Lee, \& Babin, 2008; Mittal \& Lassar, 1998; Oliver, 1999; Oliver, Rust, \& Varki, 1997; Omoregie, Addae, Coffie, Ampong, \& Ofori, 2019; Vesel \& Zabkar, 2009). O encadeamento conceitual do modelo ampliado, subsidiou a construção desta pesquisa, conforme Figura 1.

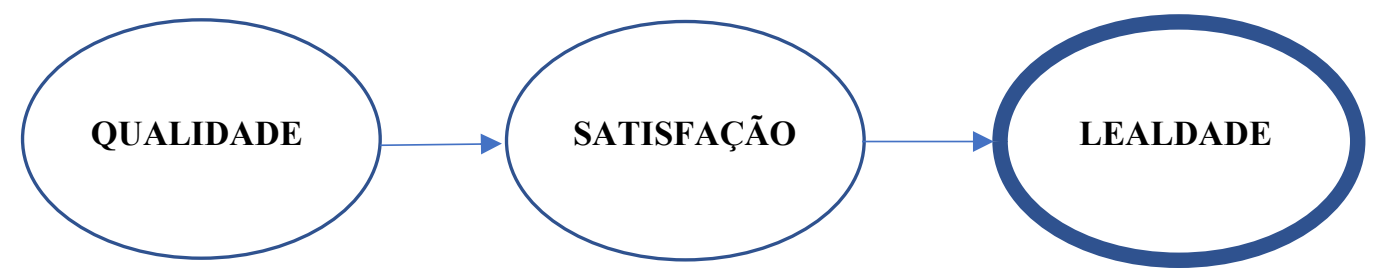

\section{Figura 1}

\section{Modelo teórico conceitual}

Fonte: Adaptado de outros estudos. Elaborado pelos autores (2019).

Para Deng et al (2010), é consenso que a satisfação é o caminho principal à fidelidade, lealdade da pessoa (Deng et al., 2010). Estudos seminais de Oliver et al (1997) e Oliver (1999), asseguram que a satisfação e a lealdade das pessoas estão intimamente interligadas; visto que a satisfação é antecedente da lealdade (Oliver, 1999; Oliver et al., 1997). 
Especialistas argumentam que o lugar onde as pessoas habitam e convivem exerce influência em sua qualidade de vida (Bougouffa \& Permana, 2018). E se o vínculo com o bairro for elevado, satisfatório, mesmo que haja insatisfação com a residência atual, os moradores tendem a se manter na área (Deane, 1990). Sendo assim, é possível destacar que as avaliações perceptivas dos residentes são oriundas da interação com características objetivas do meio em que convivem, como por exemplo o bairro (Bougouffa \& Permana, 2018; R. W. Marans, 2012).

Por outro lado, estudos apontam que a lealdade e qualidade de vida são afetadas por um conjunto de fatores, com características multidimensionais, que são influenciadas por diferentes domínios da satisfação existentes nos bairros, ambos de origem física, social, econômica, ambiental e interacional (Lovejoy, Handy, \& Mokhtarian, 2010; Sirgy \& Cornwell, 2002).

Outros estudos apontam que a satisfação com o bairro medeia a associação entre a percepção das características do lugar onde as pessoas vivem, atributos ambientais e medidas de saúde mental. Segundo esses estudos, as percepções de atributos ambientais (estética, vegetação, relações sociais, segurança, acessibilidade, infraestrutura para caminhada, acesso a serviços, uso misto da terra, conectividade com outros locais), podem influenciar a satisfação com o bairro e a saúde mental das pessoas; bem como, o excesso de ruído, tráfego intenso, violência, insegurança pode contribuir para o estresse geral e, portanto, afetar a saúde mental (Bougouffa \& Permana, 2018; Leslie \& Cerin, 2008).

\section{Método}

\section{Tipo de pesquisa}

Por tratar-se de uma pesquisa quantitativa, a tipologia adotada foi a descritiva, pois destina-se a medir e avaliar dimensões, fatos e fenômenos de determinada realidade (CRESWELL, 2010). Quanto aos procedimentos, utilizou-se pesquisa de levantamento, pois se estudou uma amostra da população buscando o conhecimento direto da realidade (CRESWELL, 2010). O método de coleta de dados foi o survey, e a análise dos dados deu-se também por meio da Structural Equation Modeling (SEM), em português, Modelagem de Equações Estruturais (MEE), em seu modelo estrutural (COSTA, 2011; CRESWELL, 2013; FLICK, 2009; HAIRJR. et al., 2010; MALHOTRA; BIRKS, 2006; YIN, 2016). A coleta de dados foi realizada através do Survey, por meio dos métodos não probabilístico, por conveniência, do tipo intencional, pois foi considerada, exclusivamente, a pessoa que ao ser consultada, aceitasse participar e concluísse por inteiro as respostas do questionário. 


\section{Locus de pesquisa}

A escolha da cidade de Natal para a realização da pesquisa deu-se pelo fato de ter ingressado na iniciativa de cidades inteligentes do IEEE Smart City, como uma cidade afiliada e a única no Brasil (IEEE, 2019). O caso de Natal, trata-se de uma abordagem de baixo para cima, com iniciativas descentralizadas e orientadas a problemas, por meio de soluções tecnológicas, visando contribuir para tornar Natal uma cidade mais inteligente, humana e melhorar a qualidade de vida de seus cidadãos (CACHO et al., 2016).

O Município de Natal está localizado no litoral oriental (leste) do Estado do Rio Grande do Norte, na Região Nordeste do Brasil, fundada em 1597, destaca-se por ser a cidade mais importante e capital do Estado. Sua área corresponde a aproximadamente 167,401 km², com população no último censo (2010) de 803.739. População estimada (2019) 884.122 pessoas, com densidade demográfica de 4.805,24 hab $/ \mathrm{km}^{2}$. Natal está dividida em quatro regiões ou zonas administrativas, que se subdividem em trinta e seis bairros (IBGE, 2019; Prefeitura Municipal do Natal, 2016).

Natal é um município $100 \%$ urbano, com importância econômica oriunda principalmente dos setores secundários e terciários, PIB de 21.845.480,68, no ranking sendo o $1^{\circ}$ do Rio Grande do Norte e o $35^{\circ}$ do Brasil, com PIB per capita de R $\$ 24.890,54$, IDHM 0,763 (IBGE, 2019). Concernente ao Índice de Bem-Estar Urbano (IBEU) local, com 0,68 (RIBEIRO; RIBEIRO, 2013).

\section{População e amostra}

No que se refere a população do estudo, foi considerado o sujeito residente na cidade de Natal-RN e maior de 18 anos. Para atender ao objetivo do estudo, procurou-se distribuir a amostra por tipo e característica urbana de bairro na região administrativa da cidade, nas zonas sul e leste. Pesquisou-se dois bairros considerados homogêneos nas referidas regiões. Na zona sul com os bairros Ponta Negra e Capim Macio; zona leste com os bairros Petrópolis e Tirol. Sendo assim, adotou-se amostra não probabilística, por conveniência, do tipo intencional (HairJr. et al., 2010).

Nessa amostra alcançou-se o quantitativo de 282 entrevistados na zona sul; 263 na zona leste; e 156 respondentes do questionário on-line, totalizando 701 residentes da cidade de Natal$\mathrm{RN}$. 


\section{Técnica de coleta de dados}

Como exposto, a respeito do tipo e estrutura empírica desta pesquisa, para a realização da coleta de dados, utilizou-se um questionário estruturado, que foi aplicado de forma impressa e presencial, por meio de abordagem direta em locais específicos dos bairros. Assim como, foi distribuído por meio eletrônico, inseridos na plataforma de acesso Google forms e aplicados através de e-mail, mensagens eletrônicas (whatsapp, etc.) e compartilhado em redes sociais (Creswell, 2013; Malhotra \& Birks, 2006).

Para isso, considerou-se a participação voluntária dos sujeitos, informando não ser necessária a identificação e que seria possível desistir da pesquisa no momento que quisessem, não ocasionando prejuízo algum para o respondente e nem para o pesquisador. Com isso, o instrumento da pesquisa foi disponibilizado, durante três meses, iniciando em 04 novembro de 2019 até o final do mês de janeiro de 2020; um tempo máximo de 30 minutos foi suficiente para que a tarefa pudesse ser realizada.

\section{Instrumento de pesquisa}

Nesta pesquisa o instrumento foi construído com base na revisão da literatura acerca das variáveis aqui propostas, referente a lealdade ao bairro. Tal questionário seguiu o método survey, composto por questões binomiais e de múltipla escolha, com utilização da escala Likert de 7 pontos, com as seguintes ponderações: no que se refere a uma medida de concordância 1 (concordo totalmente) até 7 (discordo totalmente), e/ou quanto ao grau de satisfação extremamente insatisfeito 1234567 extremamente satisfeito; e de diferencial semântico.

Tal instrumento de pesquisa foi validado por um grupo de experts, composto por profissionais que atuam na área, gestores e professores universitários. Houve a realização de um pré-teste com 50 respondentes para entendimento das questões e refinamento do instrumento de pesquisa, dos quais $100 \%$ concordaram com o instrumento proposto.

O Quadro 1 apresenta as variáveis e fontes selecionadas para compor o instrumento de pesquisa inicial, que foi alicerçada na revisão de literatura. A questão norteadora do estudo ampliado, buscou conhecer o quão satisfeitos e leais os residentes de alguns bairros estão com seu ambiente de vida e, as dimensões mais influentes na qualidade dos serviços no bairro, qual impacto desses fatores sobre a satisfação e a lealdade.

\begin{tabular}{|l|c|}
\hline VARIÁVEIS & FONTES \\
\hline
\end{tabular}




\begin{tabular}{|c|c|c|}
\hline LEALDADE & $\begin{array}{l}02 \text { Recomendaria meu bairro, como local de moradia, para } \\
\text { familiares e amigos. } \\
03 \text { Este bairro é parte de mim. Sinto-me emocionalmente } \\
\text { ligado(a). } \\
04 \text { Eu me considero um(a) morador(a) leal ao bairro. } \\
05 \text { Se eu mudasse de cidade, gostaria de morar em um bairro } \\
\text { que tivesse características similares. }\end{array}$ & $\begin{array}{l}\text { (AMERIGO, 1992) } \\
\text { (BONAIUTO et al., } \\
\text { 1999) } \\
\text { (SIRGY; } \\
\text { CORNWELL, } \\
\text { 2002) } \\
\text { (SPIERS; } \\
\text { WALKER, 2015) } \\
\text { (LESLIE; CERIN, } \\
\text { 2008) } \\
\text { (YANG, 2008) } \\
\text { (ELVAS; MONIZ, } \\
\text { 2010) } \\
\text { (QAWASMEH, } \\
\text { 2014) } \\
\text { (BOUGOUFFA; } \\
\text { PERMANA, 2018) }\end{array}$ \\
\hline
\end{tabular}

Quadro 1

Variáveis e fontes selecionadas para compor o instrumento de pesquisa inicial

Fonte: Elaborado pelos autores (2019).

\section{Análise de dados}

No que se refere a análise dos dados, foram adotadas as seguintes etapas:

A primeira etapa, referente a análise fatorial exploratória das escalas utilizadas no presente estudo, foi utilizado o software estatístico SPSS (versão 24.0); neste, além de estatísticas descritivas (média e desvio padrão, moda, frequência), realizou-se uma análise de Componentes Principais (CP), não estabelecendo rotação, pois, teoricamente, o instrumento apresenta uma originalidade em sua composição e elaboração. Para isso, considerou-se para tal análise critérios, como: o cálculo do KMO igual ou superior a 0,70 e o Teste de Esfericidade de Bartlett (qui-quadrado, $\left.\chi^{2}\right)$ significativo $(p<0,05)$ (Dancey \& Reidy, 2006; Tabachnick \& Fidell, 2001).

Os critérios de Kaiser (valor próprio igual ou superior a 1) e Cattell (distribuição gráfica dos valores próprios, visando distinguir aqueles sobressalentes) tendem a maximizar o número de fatores a extrair, por esse motivo decidiu-se efetuar uma Análise Paralela, indicada pela literatura especializada (Dancey \& Reidy, 2006; Hayton, Allen, \& Scarpello, 2004; Ledesma \& 
Valero-Mora, 2007). Neste caso, considerou-se a sintaxe do SPSS desenvolvida por O'Connor (2000) a fim de realizar a análise paralela proposta. Também, calculou-se a consistência interna (Alfa de Cronbach) dos fatores resultantes de cada escala, bem como, as correlações intraclasses (ICC) (O'connor, 2000).

Na segunda etapa, realizou-se uma confirmação da organização fatorial dos pretensos instrumentos, num segundo estudo, com nova amostra, selecionada da amostra total, realizouse uma análise fatorial confirmatória, bem como, a Modelagem de Equações Estruturais (MEE) destinadas a explicação multivariada do modelo teórico hipotetizado. Para ambos os tipos de cálculos, utilizou-se a versão 22.0 do programa AMOS Graphics para tal análise. Esse programa estatístico tem a função de apresentar, de forma mais robusta, indicadores psicométricos que visam uma melhor construção da adaptação e acurácia dos instrumentos utilizados no estudo, bem como, permite desenhar o modelo teórico pretendido no estudo. Para tanto, considerou-se como entrada a matriz de covariâncias dos itens das medidas utilizadas, o estimador $M L$ (Maximum Likelihood) (Fleury, Formiga, Souza, \& Souza, 2017). Para este tipo de análise alguns índices permitem avaliar a qualidade de ajuste dos dados ao modelo fatorial e teórico proposto (HairJr. et al., 2010; Lattin, Carroll, \& Green, 2011), devem ser considerados: O $\chi^{2} / \mathrm{g} .1$, apresentando valores até 3 para indicar um ajustamento adequado; Raiz Quadrada Média Residual (RMR); O Goodness-of-Fit Index (GFI) e o Adjusted Goodness-of-Fit Index (AGFI), assumindo valores que variam de 0 a 1 , sendo que os valores acima de 0,80 , serão satisfatórios; A Root-Mean-Square Error of Approximation (RMSEA), apresentando um intervalo de confiança de 90\% (IC90\%), devendo situar-se entre 0,05 e 0,08; Comparative Fit Index (CFI), considerando valores mais próximos de 1 como indicadores de ajustamento satisfatório; Tucker-Lewis Index (TLI), devendo variar de zero a um, com índice aceitável acima de 0,90; Expected Cross-Validation Index (ECVI) e o Consistent Akaike Information Criterion (CAIC), os quais, deverão apresentar valores baixos ao comparar a modelos alternativos.

Foi realizado o cálculo de confiabilidade composta $(\mathrm{CC})$ e da variância média extraída (VME); no primeiro indicador exige-se que o nível do escore seja acima de 0,70 , enquanto no segundo indicador é preciso um nível acima de 0,50.

\section{Resultados}

Tendo finalizada a coleta dos dados, foram inseridas as respostas dos questionários, de acordo com o que foi apresentado nos instrumentos da pesquisa, no programa Excell e transferidos posteriormente para o pacote estatístico SPSS.24.

Revista Gestão e Secretariado (GeSec), São Paulo, SP, 12(2), maio/ago., 2021, 327-352. 
Buscando atender aos objetivos pretendidos, realizou-se análises sobre a qualidade da amostra: no que se refere aos dados omissos das pesquisas (isto é, sem respostas, duplicados ou com categorias numéricas diferentes das estabelecidas nas escalas apresentadas ao respondente), observou-se um percentual abaixo do critério considerado risco $(<5 \%)$, permitindo com isso, caso existam, substituí-los pela média ou moda dos dados da amostra.

Quanto a multicolinearidade entre as variáveis, houve correlações dentro dos parâmetros definidos por Tabachnick e Fidell (2001) $[\mathrm{r} \leq 0,90$, variando de 0,05 a 0,78]. No que se refere aos outliers multivariados, através do teste de normalidade de KolmogorovSmirnov (KS), observou-se, de acordo com as orientações de Miot (2017; Nascimento, Tibana, Ferreira, \& Prestes, 2014) que os critérios atenderam a normalidade $(\mathrm{KS}=0,59)$ da amostra a um $\mathrm{p}<0,21$. Em relação Variância Comum do Método (VCM), com base no teste de um fator de Harman, seguindo a sugestão de (Podsakoff, MacKenzie, Lee, \& Podsakoff, 2003), observou-se um único fator, com autovalor acima de 1,00, tanto para a amostra total, quanto para a amostra N1 (primeira amostra) N2 (segunda amostra), um percentual, respectivamente, de $31,34 \%, 30,28 \%$ e $31,05 \%$ da covariância nas variáveis, o que implica a inexistência de um problema de variância comum do método.

Considerando que a condição da amostra não representa problemas para as análises, pois, revelou-se, a partir dos critérios estatísticos, que tal amostra atende a normalidade, podendo realizar os cálculos paramétricos destinados a avaliação da qualidade da medida e da variância dos escores médios entre as variáveis. A partir dessas condições, inicialmente, optouse em avaliar o quanto as escalas administradas no estudo são validas quanto ao seu conteúdo teórico de avaliação, resultados estes, apresentados nas próximas seções.

\section{Estudo 1 - Análise de conteúdo quanto a discriminação e representatividade dos itens}

Tendo coletado as amostras para as referidas análises, os respondentes apresentaram a seguinte caracterização sociodemográfica: $54 \%$ eram da zona sul e $46 \%$ da zona leste, a maioria (43\%) reside no bairro há mais de 11 anos, 61\% têm a casa própria, 52\% eram do sexo masculino, 57\% casados, com idade variando de 18 a 90 anos (Média $=41$ anos, d.p. =16,67); quanto ao nível de escolaridade, $52 \%$ tem graduação e 50\% tem entre 2 e 5 salários mínimos.

Com esta primeira análise estatística, verificou-se o poder discriminativo dos itens. O objetivo central deste cálculo é apresentar maior especificidade na análise na avaliação da organização distributiva dos itens nas escalas administradas aos respondentes. 
Para a realização de tal análise, tomou-se como base de avaliação os pressupostos da Teoria Clássica dos Testes (TCT); tal perspectiva refere a necessidade de avaliar se os itens são capazes de discriminar as respostas das pessoas com magnitudes próximas, considerando os grupos com pontuações dos escores inferiores e superiores em relação ao construto medido (Formiga, Fleury, Fandiño, \& Souza, 2016).

Para atender a condição hipotético-metodológica, previamente destacada no estudo quando discorrido na sua fundamentação teórica, gerou-se uma pontuação total para cada escala utilizada e em seguida, efetuou-se sua mediana, a qual, organizou estatisticamente um conjunto de escores considerado grupo inferior e grupo superior de respostas. Isto é, estabeleceu que aqueles respondentes que apresentaram pontuação abaixo da mediana foram classificados como sendo do grupo inferior e para aqueles com pontuações acima da mediana, definidos como do grupo superior (Formiga et al., 2016).

Considerando-se os itens de cada medida foi realizado um teste t de Student para amostras independentes, a fim de comparar os grupos inferiores e superiores, relacionado a discriminação de cada item para os respondentes com magnitudes próximas estatisticamente significativas. Na Tabela 1, é possível destacar que todos os itens discriminam as pontuações dos sujeitos no construto referente a escala LB (Lealdade ao Bairro). Destaca-se que todos os itens foram significativos e com médias maiores para o grupo superior, o qual, se aproxima para o grau de concordância na referida escala. Pode-se afirmar, que os respondentes reconhecem que os itens são capazes de discriminar o construto referente a LB.

Tabela 1

Poder discriminativo ( $t$ ) dos itens da escala de lealdade ao bairro

\begin{tabular}{|c|c|c|c|c|c|}
\hline \multirow{2}{*}{ Itens } & \multirow[t]{2}{*}{ Grupo discriminante } & \multirow[t]{2}{*}{ Média } & \multirow[t]{2}{*}{ d.p } & \multicolumn{2}{|c|}{ Estatística } \\
\hline & & & & $\mathrm{t}$ & p-valor \\
\hline \multirow{2}{*}{$\begin{array}{l}\text { LB1 - Se eu tivesse que mudar } \\
\text { de residência, escolheria outra } \\
\text { neste mesmo bairro. }\end{array}$} & gi & 5,10 & 1,52 & \multirow{2}{*}{$-18,11$} & \multirow{2}{*}{0,001} \\
\hline & gs & 6,87 & 0,34 & & \\
\hline \multirow{2}{*}{$\begin{array}{l}\text { LB2 - Recomendaria meu } \\
\text { bairro, como local de moradia, } \\
\text { para familiares e amigos. }\end{array}$} & gi & 5,28 & 1,31 & \multirow{2}{*}{$-18,07$} & \multirow[t]{2}{*}{0,001} \\
\hline & gs & 6,86 & 0,42 & & \\
\hline \multirow{2}{*}{$\begin{array}{l}\text { LB3 - Este bairro é parte de } \\
\text { mim. Sinto-me emocionalmente } \\
\text { ligado(a). }\end{array}$} & gi & 4,05 & 1,45 & \multirow{2}{*}{$-25,58$} & \multirow[t]{2}{*}{0,001} \\
\hline & gs & 6,61 & 0,68 & & \\
\hline \multirow{2}{*}{$\begin{array}{l}\text { LB4 - Eu me considero um(a) } \\
\text { morador(a) leal ao bairro. }\end{array}$} & gi & 4,39 & 1,50 & \multirow{2}{*}{$-22,69$} & \multirow[t]{2}{*}{0,001} \\
\hline & gs & 6,74 & 0,58 & & \\
\hline $\begin{array}{l}\text { LB5 - Se eu mudasse de cidade, } \\
\text { gostaria de morar em um bairro } \\
\text { que tivesse características } \\
\text { similares. }\end{array}$ & gs & 5,01 & 1,44 & $-19,99$ & 0,001 \\
\hline
\end{tabular}


Nota: $g s=$ grupo superior, gi $=$ grupo inferior; $\mathrm{t}=$ teste de Student, d.p. $=$ desvio padrão.

Fonte: Dados da pesquisa (2020).

Ciente de que todos os itens discriminam os itens de cada medida apresentada aos respondentes, realizou-se a avaliação da representatividade de conteúdo relativo aos escores correlacionais itens-pontuação total em cada uma das escalas. Este tipo de análise, trata de verificar a relação entre os itens e o constructo (somatório total da medida), o qual, tem como base avaliar a representação conceitual comportamento-domínio (Pasquali, 2011).

De acordo com Formiga, Fleury, Fandiño e Souza (2016), sistematicamente, através dessa análise estatística, buscou-se verificar a relação teórica apresentada nos itens da escala associados as situações especificadas neles e o quanto representam os aspectos esperados (FORMIGA et al., 2016). Com isso, a partir do cálculo da correlação de Pearson (r), verificouse a relação dos itens da escala com a pontuação total da mesma, condição a qual, espera-se que sejam significativos e tenham correlações acima de 0,50. Com isso, observou-se na Tabela 2, que as correlações item-pontuação total, da escala LB, foram positivas e significativas, bem como, acima do esperado $(>0,50)$, destacadas em negrito.

Também, é destacável a relação intra-itens em cada medida, as quais, foram altas e significativas. Isto é, estas escalas não apenas foram capazes de representar, conceitualmente, a proposta do conteúdo do item expresso na escala, bem como, revelou a existência de uma relação positiva interna entre os itens da escala. Assim, não apenas comprova a hipótese comportamento-domínio da medida desenvolvida pelos autores do estudo, mas, que, internamente, elas avaliam na mesma direção teórica do construto.

Tabela 2

Representatividade de conteúdo dos itens da LB

\begin{tabular}{|l|c|c|c|c|c|c|}
\hline Itens & LB6 & LB7 & LB8 & LB9 & LB10 & LB $_{\text {total }}$ \\
\hline LB6 & 1 & & & & & \\
\hline LB7 & $0,78^{*}$ & 1 & & & & \\
\hline LB8 & $0,63^{*}$ & $0,64^{*}$ & 1 & & & \\
\hline LB9 & $0,56^{*}$ & $0,59^{*}$ & $0,84^{*}$ & 1 & & \\
\hline LB10 & $0,73^{*}$ & $0,76^{*}$ & $0,67^{*}$ & $0,68^{*}$ & 1 & \\
\hline LB total & $\mathbf{0 , 8 4 *}$ & $\mathbf{0 , 8 5 *}$ & $\mathbf{0 , 8 9 *}$ & $\mathbf{0 , 8 6 *}$ & $\mathbf{0 , 8 8 *}$ & 1 \\
\hline
\end{tabular}

Nota: * p-valor $<0,001$

Fonte: Dados da pesquisa (2020) 
A partir desses cálculos, é possível afirmar que os sujeitos da pesquisa foram capazes de representar cognitivamente o conteúdo e sentido dos itens relacionados ao construto da lealdade ao bairro; sendo assim, procurou-se na etapa seguinte, realizar a verificação de construto a partir da análise fatorial destas medidas.

\section{Análise exploratória e de consistência interna da escala}

A partir dos resultados observados nas análises anteriores, efetuou-se uma análise fatorial exploratória. Com uma segunda amostra de 270 respondentes, os quais, todos residentes na cidade de Natal-RN e maiores de 18 anos.

Para que esta etapa fosse realizada, alguns critérios foram estabelecidos (DANCEY; REIDY, 2006): o método dos Componentes Principais (PC), deixando livre o número de fatores a que se pretende extrair, estabelecendo a rotação fatorial oblíqua (oblimin) e assumido saturação de $\pm 0,30$ para a retenção dos fatores.

Buscando obter maior segurança na tomada de decisão na escolha dos fatores, adotouse também, outros critérios de avaliação fatorial: (1) quantidade de valores próprios (eigenvalues) iguais ou superiores a 1 (Critério de Kaiser), (2) distribuição gráfica dos valores próprios, tomando como referência o ponto a partir do qual nenhum outro fator aporta consideravelmente para a estrutura (Critério de Cattell) e (3) análise paralela (Dancey \& Reidy, 2006; O’Connor, 2000).

Assim definido, realizou-se a análise fatorial exploratória para cada escala, individualmente; desta forma, foi identificado na escala LB a adequação da matriz de correlação, com um $\mathrm{KMO}=0,88$ e do Teste de Esfericidade de Bartlett, $\chi^{2} / \mathrm{gl}=2117,48 / 10, p$ $<0,001$. No critério de Cattell, foi identificado apenas um único fator na escala, tendo o seu ponto de corte apresentado valores próprios $>1,00$ para somente um fator.

Considerando o critério de Kaiser, também, observou-se que a escala LB, apresentou um único fator com valores próprios maiores que 1 (um), explicando conjuntamente $75,02 \%$ da variância total deste fator. Optou-se também, pela realização da análise paralela, assumindo os seguintes parâmetros estabelecidos no banco de dados: 270 participantes e 5 (cinco) variáveisitens, com seus valores próprios gerados em 1.000 simulações aleatórias com os itens.

Na Tabela 3, ao comparar os resultados observados nos critérios de Kaiser e na análise paralela, observou-se que aqueles resultados apresentados no critério de Kaiser foram superiores aos simulados (análise paralela); estabelece com isso, que a escala LB é unifatorial, 
pois nota-se que no segundo fator, o valor próprio (eigenvalue) foi menor do que o valor na análise paralela e, revelou um escore $>1,00(0,62)$.

Tabela 3

\section{Distribuição dos fatores da análise PC de acordo com o critério de Kaiser e análise paralela}

Fonte: Dados da pesquisa (2020).

\begin{tabular}{|c|c|}
\hline Critério de Kaiser & Análise paralela \\
\hline $\mathbf{3 , 7 5}$ & $\mathbf{1 , 2 1}$ \\
\hline 0,62 & 1,09 \\
\hline
\end{tabular}

Com base nestes critérios, realizou-se uma análise fatorial dos Componentes Principais (PC) com rotação oblíqua, eigenvalue $>1,00$ para definir o fator e saturação de $\pm 0,30$ para retenção dos itens. A utilização desta técnica se mostrou bastante adequada através dos seguintes indicadores: $\mathrm{KMO}=0,88$ e do Teste de Esfericidade de Bartlett, $\chi^{2} / \mathrm{gl}=2117,48 / 10$, $p<0,001$.

Os resultados desta fatorialização revelou uma organização unifatorial, o qual, segue o resultado encontrado nas análises para tomada de decisão empírica - valores próprios (eigenvalues) iguais ou superiores a 1 (Critério de Kaiser), distribuição gráfica dos valores próprios (Critério de Cattell) e a análise paralela.

O único fator, definido como Lealdade ao Bairro, conceituado como: um estado psicológico do morador que está satisfeito com o bairro e é leal ao mesmo, que deseja continuar residindo no ambiente e o recomendaria a familiares e amigos. Explicou conjuntamente $75,02 \%$ da variância do fator, composto pelos itens: LB06: Se eu tivesse que mudar de residência, escolheria outra neste mesmo bairro; LB07: Recomendaria meu bairro, como local de moradia, para familiares e amigos; LB08: Este bairro é parte de mim. Sinto-me emocionalmente ligado(a); LB09: Eu me considero um(a) morador(a) leal ao bairro; LB10: Se eu mudasse de cidade, gostaria de morar em um bairro que tivesse características similares (Tabela 4).

Tabela 4

Análise dos principais componentes (PC) da LB

\begin{tabular}{|c|c|c|}
\hline Itens & $\mathrm{a}_{\mathrm{if2}}$ & $\mathrm{h}^{2}$ \\
\hline LB06 & 0,88 & 0,73 \\
\hline LB07 & 0,87 & 0,76 \\
\hline
\end{tabular}




\begin{tabular}{|c|c|c|}
\hline LB08 & 0,87 & 0,76 \\
\hline LB09 & 0,85 & 0,72 \\
\hline LB10 & 0,85 & 0,79 \\
\hline Número total de itens & 05 \\
\hline Valores próprios & 3,75 \\
\hline Variância Explicada & $71,02 \%$ \\
\hline Alpha de Chronbach $(\alpha)$ & 0,91 \\
\hline Alpha de Chronbach $(\alpha)[$ item & $0,88-0,90$ \\
\hline exclú́do] & \multicolumn{2}{|c|}{0,91} \\
\hline ICC & \multicolumn{2}{|c|}{$(0,90-0,94)$} \\
\hline Média (dp) & $28,56(6,55)$ \\
\hline
\end{tabular}

Nota: $\mathrm{a}_{\mathrm{ifl}}=$ Carga Fatorial; $\mathrm{h}^{2}=$ Cumunalidade.

Fonte: Dados da pesquisa (2020).

$\mathrm{Na}$ Tabela 4, é apresentado o conteúdo de cada item, sua saturação (carga fatorial) e comunalidade, os indicadores de consistência interna (Alfa de Cronbach) e o ICC, e variância explicada pelo fator; destaca-se que todos os indicadores estiveram dentro do padrão psicométrico exigido (HairJr. et al., 2010; Pasquali, 2011), o que garante a qualidade fatorial da escala LB.

\section{Análise fatorial confirmatória da escala LB}

Nesta etapa do resultado, com base nos indicadores psicométricos da análise fatorial exploratória observado na escala LB, efetuou-se o cálculo da análise fatorial confirmatória (AFC). Para isso, empregou-se o pacote estatístico AMOS 22.0, tendo como orientação hipotética a representação de um construto reflexivo, o qual, tem seu núcleo de base conceitual associado aos itens, assumindo a organização fatorial apresentada na análise exploratória, tendo a proposta fatorial observada na análise fatorial, apresentada na Tabela 4, a qual, espera-se ser confirmada.

Uma terceira coleta foi realizada, destinada a aplicação deste cálculo, esta, apresentou características muito próximas a N1. Com isso, 275 respondentes, todos eram residentes na cidade de Natal-RN e maiores de 18 anos. Para uma leitura mais fluida dos modelos estruturais, 
a análise foi realizada individualmente, para cada escala. Efetuou-se o cálculo, deixando livre as covariâncias (phi, $\varphi$ ), fixando o modelo de estimação da discrepância através do método Maximum Likelihood (ML), condição a qual, permitiu observar indicadores de qualidade de ajuste para o modelo unifatorial e que revelou indicadores psicométricos próximos as recomendações apresentadas na literatura (Byrne, 1989; Tabachnick \& Fidell, 2001; Van de Vijver \& Leung, 1997).

É preciso destacar que o uso do método Maximum Likelihood (ML), foi utilizado, tanto por ser um método bastante frequente na $\mathrm{AFC}$, quanto por avaliar construtos originais com escalas contínuas, bem como, devido a capacidade deste método, de acordo com Harrington (HairJr., Anderson, Tatham, \& Black, 2009) este estimador fornece erros padrões para cada parâmetro estimado, com teste de significância e precisão dos intervalos de confiança, contempla a função de ajustes de erro com o objetivo de verificar índices de qualidade desses ajustes para garantir de forma mais consistente o modelo estrutural proposto.

E ainda, de acordo com Brown (2015), o ML tem alguns pressupostos que serão úteis, por exemplo: tal procedimento deverá ser utilizado com grandes tamanhos amostrais (isto é, o 'n' amostral acima de 100 sujeitos) (Brown, 2015; HairJr. et al., 2010), todas as variáveis devem ser contínuas, as variáveis devem ter distribuição normal multivariada ou mesmo a normalidade univariada e os outliers. Critérios estes, contemplados neste estudo.

Destaca-se que o modelo pretendido (modelo unifatorial) apresentou indicadores estatísticos que garantiram a estrutura fatorial da LB, observada anteriormente na análise exploratória (conferir Tabela 4), apresentando as seguintes razões psicométricas: modelo unifatorial não ajustado $\left[\chi^{2} / \mathrm{gl}=20,35, \mathrm{RMR}=0,12, G F I=0,88, A G F I=0,64, C F I=0,90\right.$, TLI $=0,81, \operatorname{RMSEA}(90 \% I C)=0,27(0,22-0,31), \mathrm{CAIC}=167,94$ e ECVI $=0,44 ;$ em relação ao

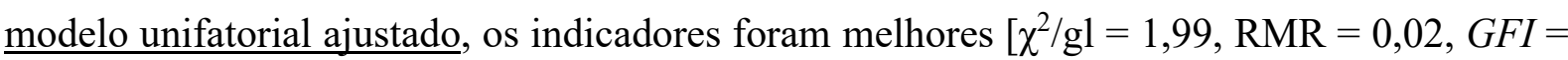
$0,99, A G F I=0,98, C F I=1,00, \mathrm{TLI}=1,00, \operatorname{RMSEA}(90 \% I C)=0,01(0,00-0,11), \mathrm{CAIC}=88,01$ e $\operatorname{ECVI}=0,10]$.

Também, consideraram-se os indicadores AIC, BIC e BCC, os quais, garantiram o modelo unifatorial ajustado. Observou-se que o modelo ajustado, apresentou o seguinte indicador: $\mathrm{AIC}=27,99, \mathrm{BIC}=70,01$ e $\mathrm{BCC}=28,56$; enquanto o modelo não ajustado, foi: $\mathrm{AIC}$ $=121,76, \mathrm{BIC}=157,94$ e $\mathrm{BCC}=122,21$. É destaque que os indicadores do modelo pretendido foram muito melhores, pois, exige-se que nestes critérios, quanto menor for o valor, melhor a segurança empírica do modelo.

As saturações (Lambdas, $\lambda$ ) estiveram dentro do intervalo esperado $\mid 0$ - $1 \mid$, sem problemas da estimação empírica. Destaca-se também, que todas as saturações foram 
estatisticamente diferentes de zero $(t>1,96, p<0,05)$ comprovando a existência do modelo unifatorial ajustado (Tabela 5).

Tabela 5

Estrutura Fatorial da LB

\begin{tabular}{c|c|c|c|c|c}
\hline \multirow{2}{*}{$\begin{array}{c}\xi \\
\text { (Constructo) }\end{array}$} & $\begin{array}{c}\chi \\
\text { (Variáveis) } \\
{[\text { itens }]}\end{array}$ & $\begin{array}{c}\lambda \\
\text { (Escores } \\
\text { Associativos) }\end{array}$ & $\begin{array}{c}\mathrm{E} \\
\text { (erros) }\end{array}$ & CC & VME \\
\hline \multirow{4}{*}{ LB } & LB 6 & 0,84 & 0,71 & & \\
\cline { 2 - 7 } & LB 7 & 0,85 & 0,73 & & \\
\cline { 2 - 7 } & LB 8 & 0,80 & 0,63 & 0,93 & 0,61 \\
\cline { 2 - 7 } & LB 9 & 0,78 & 0,61 & & \\
\cline { 2 - 7 } & LB 10 & 0,85 & 0,72 & & \\
\hline
\end{tabular}

Nota: $\lambda=$ Escores fatoriais da estrutura; $\varepsilon$ (erros) $=$ Erros de medida da estrutura; $\mathrm{X}=$ variáveis (itens); $\xi=$ Construto VME = Variância Média Extraída. $\mathrm{CC}=$ Confiabilidade Composta. $\mathrm{LB}=$ Lealdade ao Bairro Fonte: Dados da pesquisa (2020).

Em relação à validade do construto em questão, efetuou-se tanto o cálculo de confiabilidade composta (CC) quanto da variância média extraída (VME), observou-se, respectivamente, indicadores de 0,93 e 0,61. Estes evidenciam a confiabilidade e validade convergente do construto, o qual, é adequado a estrutura fatorial da referida medida na amostra.

Esses resultados foram confirmados a partir dos observados das estimativas de predição. Com base na análise de regressão, identificou-se uma razão critério para as variáveis, que tanto estavam no intervalo estatisticamente exigido, quanto foram diferentes de zero $(t>1,96, p<$ $0,05)$ e significativos (Tabela 6).

Tabela 6

Indicadores das estimativas preditivas da associação itens-fator da LB

\begin{tabular}{c|c|c|c|c|c|c}
\hline Itens & Interação & Construto & Estimativa & d.p. & $\begin{array}{c}\text { Razão } \\
\text { Critério }\end{array}$ & p-valor \\
\hline LB01 & $<---$ & LB & 1,000 & --- & --- & --- \\
\hline LB02 & $<---$ & LB &, 875 &, 050 & 17,367 & 0,001 \\
\hline LB03 & $<---$ & LB & 1,081 &, 069 & 15,572 & 0,001 \\
\hline LB04 & $<---$ & LB & 1,012 &, 067 & 15,214 & 0,001 \\
\hline LB05 & $<---$ & LB & 1,013 &, 059 & 17,166 & 0,001 \\
\hline
\end{tabular}

Fonte: Dados da pesquisa (2020).

Considerando que a referida medida apresentou indicadores de acordo com o que é exigido na literatura estatística e de psicometria, os quais, foram significativos; pode-se com 
isso, confirmar que a estrutura fatorial do construto apresentou confiabilidade em sua mensuração, condição que permitiu avaliar o objetivo central do artigo em distintos bairros da cidade de Natal-RN.

Considerando que o modelo fatorial proposto foi confirmado e sua estrutra unifatorial, pois, tanto indicadores estatísticos quanto escores Lambdas foram adequados e significativos. A partir desse modelo fatorial, optou-se em realizar análises estatisticas de comparações (teste t de Studente e ANOVA) a fim de avaliar as diferenças nas pontuações médias nas variáveis dependentes versus independentes.

Desta maneira, a partir de um teste $t$ de Student, comparou a pontuação média do respondente da zona leste e sul em relação ao construto da Lealdade ao bairro (LB). Na Tabela 7, pode-se observar que houve difença entre as médias apenas no DQSB na zona leste; os demais construtos não se diferenciaram entre essas zonas.

Tabela 7

Diferenças entre as médias em relação a lealdade ao bairro em função da zona sul e leste.

\begin{tabular}{c|c|c|c|c|c|c}
\hline \multirow{2}{*}{ Construtos } & \multirow{2}{*}{ ZONA } & \multirow{2}{*}{ Média } & \multirow{2}{*}{ d.p. } & \multicolumn{3}{|c}{ Estatística } \\
\cline { 7 - 8 } & & & & $\mathrm{t}$ & $\mathrm{p}$-valor & $95 \% \mathrm{IC}$ \\
\hline \multirow{2}{*}{ LB } & Sul & 28,69 & 6,68 & & & \multirow{2}{*}{0,49} \\
\cline { 2 - 4 } & Leste & 28,41 & 6,42 & & 0,62 & $-0,82$ a 1,38 \\
\hline
\end{tabular}

Fonte: Dados da pesquisa (2020).

A partir desses resultados, chama-se atenção para a distribuição dos bairros nas zonas; estas, eram distribuídas em dois bairros, cada (zona sul - Ponta Negra e Capim Macio; zona leste - Petrópolis e Tirol). Com isso, realizou-se o cálculo da Anova one-way, associada ao teste post-hoc de Scheffé, a qual, revelou que os escores médios, em nenhum bairro, foi significativo; isto é, ao considerá-los, não faz diferença a Lealdade ao bairro (LB) para os respondentes (Tabela 8).

Tabela 8

Diferenças entre as médias em relação a lealdade ao bairro em função dos bairros

\begin{tabular}{c|c|c|c|c|c|c|c|c}
\hline Construto & Bairros & Média & d.p. & \multicolumn{2}{|c|}{$95 \%$ IC } & \multicolumn{3}{|c|}{ Estatística } \\
\cline { 5 - 9 } & & & & Baixo & Alto & $\begin{array}{c}\text { F } \\
\text { Friedman }\end{array}$ & gl & p-valor \\
\hline \multirow{2}{*}{ LB } & PN & 28,68 & 7,01 & 27,43 & 29,93 & 2,16 & 3 & 0,09 \\
\cline { 3 - 6 } & CM & 28,62 & 6,43 & 27,68 & 29,70 & & \multirow{2}{*}{} \\
\hline
\end{tabular}




\begin{tabular}{c|c|c|c|c|c|c|c|c}
\hline & PETRO & 29,37 & 6,39 & 28,29 & 30,46 & \multirow{4}{*}{} & & \\
\cline { 2 - 6 } & TIR & 27,36 & 6,30 & 26,25 & 28,47 & & \\
\cline { 2 - 6 } & Total & 28,55 & 6,55 & 28,00 & 29,10 & & & \\
\hline
\end{tabular}

Nota: $\mathrm{PN}=$ Ponta Negra; $\mathrm{CM}=$ Capim Macio; PETRO $=$ Petrópolis; TIR $=$ Tirol.

Fonte: Dados da pesquisa (2020).

\section{Discussão}

A partir desses resultados, é preciso destacar que a escala relativa à lealdade ao bairro é consistente e que, com base em seus indicadores psicométricos é capaz de mensurar a lealdade; que neste estudo define-se como um estado psicológico do morador que está satisfeito com o bairro e é leal ao mesmo, que deseja continuar residindo no ambiente e o recomendaria a familiares e amigos. Seja ao avaliar a análise de conteúdo dos itens, seja na análise exploratória e posteriormente, na confirmatória, a escala é confiável e fidedigna (PASQUALI, 2011; HairJr. et al., 2010).

Tais resultados corroboram relação causal entre qualidade, satisfação e lealdade, onde a qualidade tem relação direta e positiva com a satisfação, e esta tem relação direta e positiva com a lealdade (Cronin Jr \& Taylor, 1992; Fitzsimmons \& Fitzsimmons, 2014; Gronroos, 1984; Parasuraman et al., 1985).

De acordo com este estudo e conforme relações causais observadas na revisão de literatura, estruturou-se o seguinte resumo com os achados teóricos e suas implicações positivas. Quanto a argumentação de que a qualidade dos serviços oferecidos no bairro influencia positivamente à lealdade de seus moradores; apresenta sustentação teórica nos seguintes estudos relacionados a Teoria dos Serviços, onde fundamenta que Qualidade implica positivamente com Lealdade (Chang \& Thai, 2016; Chen \& Cheng, 2012; Cronin Jr \& Taylor, 1992; Harris \& Goode, 2004; Omoregie et al., 2019; Singla, 2012).

Quanto a argumentação de que a satisfação dos moradores com os serviços oferecidos no bairro influencia a lealdade de seus moradores com o bairro, apresenta sustentação teórica nos seguintes estudos relacionados a Teoria dos Serviços, onde fundamenta que Satisfação implica positivamente com Lealdade (Ahrholdt et al., 2016; Caceres \& Paparoidamis, 2007; Chang \& Thai, 2016; Chen \& Cheng, 2012; Deng et al., 2010; Harris \& Goode, 2004; Kassim \& Abdullah, 2010; Lai et al., 2009; Lee et al., 2008; Oliver, 1999; Oliver et al., 1997; Omoregie et al., 2019; Vesel \& Zabkar, 2009).

E na Teoria da Satisfação Residencial, onde fundamenta que Satisfação dos Moradores com Bairro, implica positivamente com Lealdade com o Bairro (Bonaiuto, Aiello, Perugini, 
Bonnes, \& Ercolani, 1999; Qawasmeh, 2014). Em uma análise mais abrangente, tem-se que Teoria da Satisfação Residencial fundamenta que a Satisfação com o Bairro, a Habitação e com a Vida (necessidades residenciais atendidas), implicam positivamente à Permanência no Bairro (Basolo \& Strong, 2002; Deane, 1990; Galster, 1987; Schwanen \& Mokhtarian, 2004; Speare Jr, 1974).

Segundo Chang e Thai (2016), a lealdade origina-se da relação de qualidade do serviço e da satisfação, ambas exercem um impacto direto e positivo sobre a lealdade do cliente. Essa fidelização faz com que o indivíduo desenvolva um compromisso consistente e queira repetir a experiência.

\section{Conclusões}

Os resultados revelaram que a escala de Lealdade ao Bairro (LB) é consistente e fidedigna para uma estrutura fatorial de um único fator e que não houve diferença entre as zonas urbanas pesquisadas, comprovando com isso, o mesmo nível de lealdade.

Assim, na perspectiva da gestão urbana e qualidade dos serviços, vislumbrando melhores condições de qualidade de vida aos cidadãos nos bairros e nas cidades, este estudo propõe uma ferramenta de mensuração da lealdade de morados para com seus bairros, possibilitando análises que poderão direcionar a tomada de decisão por parte dos gestores, favorecendo bairros mais atraentes, acolhedores, com bem-estar e oportunidades às pessoas.

A recomendação para futuros estudos, é ampliar a área da pesquisa abrangendo as outras zonas que são norte e oeste, propiciando uma análise comparativa, pois são áreas com perfil sociodemográfico distinto das abordadas neste estudo.

\section{Referências}

Ahrholdt, D. C., Gudergan, S. P., \& Ringle, C. M. (2016). Enhancing Service Loyalty: The Roles of Delight, Satisfaction, and Service Quality. Journal of Travel Research, 1-15. https://doi.org/10.1177/0047287516649058

Amerigo, M. (1992). A Model of Residential Satisfaction. Conference Paper. Madrid, 1992. Amerigo, M., \& Aragones, J. I. (1997). A theoretical and methodological approach to the study of residential satisfaction. Journal of Environmental Psychology, (17), 47-57. https://doi.org/10.1006/jevp.1996.0038 
Basolo, V., \& Strong, D. (2002). Understanding the neighborhood: From residents' perceptions and needs to action. Housing Policy Debate, 13(1), 83-105. https://doi.org/10.1080/10511482.2002.9521436

Bonaiuto, M., Aiello, A., Perugini, M., Bonnes, M., \& Ercolani, A. P. (1999). Multidimensional perception of residential environment qualityand neighbourhood attachment in the urban Environment. Journal of Environmental Psychology, 19, 331-352. https://doi.org/10.1016/j.ijinfomgt.2009.10.001

Bougouffa, I., \& Permana, A. S. (2018). A Study on the Linkages between Residential Satisfaction and the Overall Quality of Life in Bandar Tun Razak Area of Kuala Lumpur City, Malaysia. Applied Research Quality Life, 991-1013. https://doi.org/https://doi.org/10.1007/s11482-017-9570-3

Bramley, G., Dempsey, N., Power, S., Brown, C., \& Watkins, D. (2009). Social sustainability and urban form: evidence from five British cities. Environment and Planning, 41, 2125 2143. https://doi.org/10.1068/a4184

Brown, T. A. (2015). Methodology in the social sciences. Confirmatory factor analysis for applied research. The Guilford Press.

Byrne, B. M. (1989). A primer of LISREL: Basic applications and programming for confirmatory factor analytic models. (Springer-Verlag, Org.). New York: SpringerVerlag.

Caceres, R. C., \& Paparoidamis, N. G. (2007). Service quality, relationship satisfaction, trust, commitment and business-to-business loyalty. European Journal of Marketing, 41(7/8), 836-867. https://doi.org/10.1108/03090560710752429

Cacho, N., Lopes, F., Cavalcante, E., \& Santos, I. (2016). A smart city initiative: The case of Natal. IEEE 2nd International Smart Cities Conference: Improving the Citizens Quality of Life, ISC2 2016 - Proceedings. https://doi.org/10.1109/ISC2.2016.07580774

Chang, C., \& Thai, V. V. (2016). Do port security quality and service quality influence customer satisfaction and loyalty? Maritime Policy \& Management, 1-17. https://doi.org/10.1080/03088839.2016.1151086

Chen, C., \& Cheng, L. (2012). A study on mobile phone service loyalty in Taiwan. Total Quality Management \& Business Excellence, 23(7), 807-819. https://doi.org/10.1080/14783363.2012.661129

Creswell, J. W. (2013). Pesquisa de métodos mistos: métodos qualitativos, quantitativos e misto (Penso). Porto Alegre - RS: Penso.

Cronin Jr, J. J., \& Taylor, S. A. (1992). Measuring Service Quality - A Reexamination And Revista Gestão e Secretariado (GeSec), São Paulo, SP, 12(2), maio/ago., 2021, 327-352. 
Extension. Journal of Marketing, 56(3), 55-68. https://doi.org/10.2307/1252296

Dancey, C. P., \& Reidy, J. (2006). Estatística sem matemática para psicologia: usando SPSS para Windows (Artmed). Porto Alegre: Artmed.

Dassopoulos, A., Batson, C. D., Futrell, R., \& Brents, B. G. (2012). Neighborhood Connections, Physical Disorder, and Neighborhood Satisfaction in Las Vegas. Urban Affairs Review, 48(4), 571-600. https://doi.org/10.1177/1078087411434904

Deane, G. D. (1990). Mobility and Adjustments: Paths to the Resolution of Residential Stress. Demography, 27(1), 65-66.

Deng, Z., Lu, Y., Kee, K., \& Zhang, J. (2010). Understanding customer satisfaction and loyalty: An empirical study of mobile instant messages in China. International Journal of Information Management, 30(4), 289-300. https://doi.org/10.1016/j.ijinfomgt.2009.10.001

Elvas, S., \& Moniz, J. M. V. (2010). Sense of community, quality and life satisfaction. Análise Psicológica, 3(XXVIII), 451-464.

Fitzsimmons, J. A., \& Fitzsimmons, M. J. (2014). Administração de Serviços: operações, estratégia e tecnologia da informação. (7 $7^{\mathrm{a}}$ ed., Org.) (AMGH). Porto Alegre.

FORMIGA, N. S., FLEURY, L. F. O., FANDIÑO, A. M., \& SOUZA, M. A. (2016). Evidência empírica de uma medida da anomia organizacional em trabalhadores brasileiros. Revista de Psicologia da UCV, 18(1), 43-59. https://doi.org/10.18050/revpsi.v18n1a4.2016

Fornara, F., Bonaiuto, M., \& Bonnes, M. (2010). Cross-Validation of Abbreviated Perceived Residential Environment Quality (PREQ) and Neighborhood Attachment (NA) Indicators. Environment and Behavior, 42(2), 171-196. https://doi.org/10.1177/0013916508330998

Galloway, S. (2006). Quality of life and well-being: measuring the benefits of culture and sport.

A literature review. In Social Research (p. 4-97). Scottish Executive.

Galster, G. (1987). Identifying the correlates of dwelling satisfaction an empirical critique. Environment and Behavior, 19(5), 539-568. https://doi.org/10.1177/0013916587195001 Grogan-kaylor, A., Woolley, M., Mowbray, C., Msw, T. M. R., Gilster, M., Karb, R., ... Alaimo, K. (2006). Predictors of Neighborhood Satisfaction. Journal of Community Practice, 14(4), 27-50. https://doi.org/http://dx.doi.org/10.1016/j.jclepro.2015.09.001

Gronroos, C. (1984). A Service Quality Model and its Marketing. European Journal of Marketing, 18(4), 36-44.

Hadavi, S., Kaplan, R., \& Hunter, M. R. (2018). How does perception of nearby nature affect multiple aspects of neighbourhood satisfaction and use patterns? Landscape Research, 43(3), 360-379. https://doi.org/10.1080/01426397.2017.1314453

Revista Gestão e Secretariado (GeSec), São Paulo, SP, 12(2), maio/ago., 2021, 327-352. 
HairJr., Anderson, R. E., Tatham, R. L., \& Black, W. C. (2009). Análise multivariada de dados. (Bookman, Org.) (5a . ed.). Porto Alegre - RS: Bookman.

HairJr., Black, W. C., Babin, B. J., \& Anderson, R. E. (2010). Multivariate Data Analysis. (P. H, Org.) (Prentice H). New Jersey, 2010: 7. ed.

Harris, L. C., \& Goode, M. M. H. (2004). The four levels of loyalty and the pivotal role of trust: a study of online service dynamics. Journal of Retailing, 80, 139-158. https://doi.org/10.1016/j.jretai.2004.04.002

Ibem, E. O., \& Aduwo, E. B. (2013). Assessment of residential satisfaction in public housing in Ogun State, Nigeria. Habitat International, 40, 163-175. https://doi.org/10.1016/j.habitatint.2013.04.001

Jansen, S. J. T. (2014). The impact of the have-want discrepancy on residential satisfaction. Journal of Environmental Psychology, 1-41. https://doi.org/10.1016/j.jenvp.2014.04.006

Kassim, N., \& Abdullah, N. A. (2010). The effect of perceived service quality dimensions on customer satisfaction, trust, and loyalty in e-commerce settings: A cross cultural analysis. Asia Pacific Journal of Marketing and Logistics, 22(3), 351-371. https://doi.org/10.1108/13555851011062269

Khaef, S., \& Zebardast, E. (2015). Assessing Quality of Life Dimensions in Deteriorated Inner Areas: A case from Javadieh Neighborhood in Tehran Metropolis. Social Indicators Research, (13). https://doi.org/10.1007/s11205-015-0986-6

Lai, F., Grif, M., \& Babin, B. J. (2009). How quality, value, image, and satisfaction create loyalty at a Chinese telecom. Journal of Business Research, 62, 980-986. https://doi.org/10.1016/j.jbusres.2008.10.015

Lattin, J., Carroll, J. D., \& Green, P. E. (2011). Análise de dados multivariados (Cengage Le). São Paulo: Cengage Le.

Lee, Y., Lee, C., Lee, S., \& Babin, B. J. (2008). Festivalscapes and patrons' emotions, satisfaction, and loyalty. Journal of Business Research, 61, 56-64. https://doi.org/10.1016/j.jbusres.2006.05.009

Leslie, E., \& Cerin, E. (2008). Are perceptions of the local environment related to neighbourhood satisfaction and mental health in adults? Preventive Medicine, 47, 273278. https://doi.org/10.1016/j.ypmed.2008.01.014

Lovejoy, K., Handy, S., \& Mokhtarian, P. (2010). Neighborhood satisfaction in suburban versus traditional environments: An evaluation of contributing characteristics in eight California neighborhoods. Landscape and Urban Planning, 97(1), 37-48. https://doi.org/10.1016/j.landurbplan.2010.04.010 
Malhotra, N. K., \& Birks, D. F. (2006). Marketing research: an applied approach. (P. Hall, Org.) (Rev. 2nd E). Prentice Hall.

Marans, R., \& Rodgers, W. (1975). Toward an understanding of community satisfaction. In Metropolitan America in Contemporary Perspective (p. chapter 7). New York: Halstead Press.

Marans, R. W. (2012). Quality of Urban Life Studies: An Overview and Implications for Environment-Behaviour Research. Procedia - Social and Behavioral Sciences, 35, 9-22. https://doi.org/10.1016/j.sbspro.2012.02.058

Marôco, J. (2010). Analise de equações estruturais: fundamentos teóricos, software \& aplicações (ReportNumb). Pêro Pinheiro: ReportNumber.

Mesch, G. S., \& Manor, O. (1998). Social ties, environmental perception, and local attachment. Environment and Behavior, 504-519. https://doi.org/10.1177/001391659803000405

Mittal, B., \& Lassar, W. M. (1998). Why do customers switch? The dynamics of satisfaction versus loyalty. Journal of Services Marketing, 12(3), 177-194. https://doi.org/10.1108/08876049810219502

Momtaz, Y. A., Fallahi, B., \& Delbari, A. (2018). Residential Satisfaction Among Iranian Senior Citizens. The Open Psychology Journal, 11, 89-94. https://doi.org/10.2174/1874350101811010089

O'Connor, B. P. (2000). SPSS and SAS programs for determining the number of components using parallel analysis and Velicer's MAP test. Behavior Research Methods, instruments, and computers, 32(3), 396-402.

Oliver, R. L. (1999). Whence Consumer Loyalty? Journal of Marketing, 63, 33-44.

Oliver, R. L., Rust, R. T., \& Varki, S. (1997). Customer delight Foundations, findings, and managerial insight. Journal of Retailing, 73(3), 311-336.

Omoregie, O. K., Addae, J. A., Coffie, S., Ampong, G. O. A., \& Ofori, K. S. (2019). Factors influencing consumer loyalty: evidence from the Ghanaian retail banking industry. International Journal of Bank Marketing. https://doi.org/10.1108/IJBM-04-2018-0099

Parasuraman, A., Zeithaml, V. A., \& Berry, L. L. (1985). A conceptual Model of Service Quality and its Implications for Future Research. Journal of Marketing, 49, 41-50.

Pasquali, L. (2011). Psicometria: teoria dos testes na psicologia e na educação. (Vozes, Org.) (4⿳a .ed.). Petrópolis - RJ: Vozes.

Podsakoff, P. M., MacKenzie, S. B., Lee, J.-Y., \& Podsakoff, N. P. (2003). Common method biases in behavioral research: A critical review of the literature and recommended remedies. Journal of Applied Psychology, 88(5), 879-903. https://doi.org/10.1037/0021- 
9010.88 .5 .879

Qawasmeh, R. (2014). Identification of the quality of urban life assessment aspects in residential neighbourhoods in Doha. The Sustainable City, 191(IX), 391-402. https://doi.org/10.2495/SC140331

Riazi, M., \& Emami, A. (2018). Residential satisfaction in a ff ordable housing: A mixed method study. Cities, 82, 1-9. https://doi.org/10.1016/j.cities.2018.04.013

Ribeiro, L. C. de Q., \& Ribeiro, M. G. (2013). Ibeu: Índice de Bem-Estar Urbano. (L. Capi, Org.) (Letra Capi). Rio de Janeiro, 2013: Letra Capi.

Riecken, G., Shemwell, D., \& Yavas, U. (2000). Assessing a Place to Live: A Quality of Life Perspective. Journal of Nonprofit \& Public Sector Marketing, 7(2), 17-29. https://doi.org/10.1300/J054v07n02

Schwanen, T., \& Mokhtarian, P. L. (2004). The extent and determinants of dissonance between actual and preferred residential neighborhood type. Environment and Planning B: Planning and Design, 31, 759-784. https://doi.org/10.1068/b3039

Singla, V. (2012). Role of service quality and customer satisfaction in predicting customer loyalty. J. Global Business Advancement, 5(3), 248-259.

Sirgy, M. J., \& Cornwell, T. (2002). How neighborhood features affect quality of life. Social Indicators Research, 59(1), 79-114. https://doi.org/10.1023/A:1016021108513

Smrke, U., Blenkuš, M., \& Sočan, G. (2018). Vprašalniki zadovoljstva z bivalnim okoljem: sistematični pregled. Urbani Izziv, 29(2), 7-21. https://doi.org/10.5379/urbani-izziv-201829-02-001

Speare Jr, A. (1974). Residential Satisfaction as an Intervening. DEMOGRAPHV, 11(2), 173188.

Tabachnick, B. G., \& Fidell, L. S. (2001). Using Multivariate Statistics. (4. Ed., Org.) (Allyn and). Boston: Allyn and.

Van de Vijver, F., \& Leung, K. (1997). Methods and data analysis for cross-cultural research. (Sage, Org.) (Sage). Thousand Oaks: Sage.

Vesel, P., \& Zabkar, V. (2009). Managing customer loyalty through the mediating role of satisfaction in the DIY retail loyalty program. Journal of Retailing and Consumer Services, 16(5), 396-406. https://doi.org/10.1016/j.jretconser.2009.05.002

Weidemann, S. U. E., \& Anderson, J. R. (1985). A Conceptual Framework for Residential Satisfaction. Home Environments ( $\left.8^{\circ} \mathrm{ed}\right)$. New York: Springer Science, Business Media New York.

Yang, Y. (2008). A Tale of Two Cities: Physical Form and Neighborhood Satisfaction in Revista Gestão e Secretariado (GeSec), São Paulo, SP, 12(2), maio/ago., 2021, 327-352. 
Metropolitan Portland and Charlotte. Journal of the American Planning Association, 4363, 307-323. https://doi.org/10.1080/01944360802215546

Submetido em: 30.11 .2020

Aceito em: $\quad 31.05 .2021$ 